\title{
Comparison of eosinophil counts in patients with acute pulmonary embolism: Could it be a predictor factor?
}

\author{
Asli Kurtar Mansiroglu, Mehmet Cosgun, Isa Sincer, Yilmaz Gunes \\ Department of Cardiology, Bolu Abant Izzet Baysal University, School of Medicine, Bolu, Turkey
}

\section{ABSTRACT}

Aim: To investigate whether there is a relationship between both massive and sub-massive pulmonary embolism (PE) and eosinophil counts in order to evaluate it as a predictor factor.

Methods: This retrospective study included 108 patients (64 sub-massive and 44 massive) who received both tomographic and clinical diagnoses of pulmonary embolism, and 75 subjects served as controls. Hemogram parameters were compared between patients with massive and sub-massive pulmonary embolism and those of control groups.

Results: In terms of white blood cell and eosinophil counts, the lowest value was evident in the massive PE group whereas the control group had the highest value. The eosinophil counts increased significantly one week after the treatment when compared to those at the presentation with PE $(0.112(0.003-0.853) v s$. $(0.144$ (0.011$0.914), p=0.01)$. Spearman correlation test showed a significant positive correlation between right ventricular dysfunction or elevated cardiac troponin level and massive PE $(r=0.54, p<0.001)$, whereas a negative correlation was detected between eosinophil count and the presence of massive $\mathrm{PE}(r=-0.36, p<0.001)$.

Conclusion: The results of our study suggest that lower eosinophil counts may lead a physician to suggest a higher probability of acute massive pulmonary embolism rather than sub-massive pulmonary embolism. However, further randomized studies are required to confirm these findings.

Key words: Pulmonary embolism, acute disease, eosinophil count, predictive value.

$\triangle$ Dr. Asli Kurtar Mansiroglu

Department of Cardiology, Bolu Abant Izzet Baysal

University, School of Medicine, Bolu, Turkey

E-mail: dr.asli.kurtar@gmail.com

Received: 2021-04-26

Accepted: 2021-07-25 / Published online: 2021-10-01

\section{Introduction}

Pulmonary embolism (PE) is characterized by an occlusion of pulmonary arteries. Acute PE is a life-threatening condition that needs immediate treatment to prevent death [1]. The severity and prognosis of PE depend on the involvement degree of the pulmonary arterial bed [2,3].
Identification of predisposing factors and markers for PE is crucial for determining the treatment modality. Eosinophils are the 2nd largest member of the leukocyte family [4]. They have been associated with allergic diseases, parasitic infections, autoimmune diseases, and myelodysplastic syndrome [4]. In addition, evidence has shown that eosinophils are associated with stent thrombosis, vasospastic angina, coronary artery disease, and coronary collateral development [4-6]. Eosinophils are known to comprise many granules facilitating both the formation and growth of thrombus in several diseases $[7,8]$. 
However, the literature lacks data from the comparison of eosinophil counts between patients with massive and sub-massive PE. Strong vasoactive and pro-coagulant effects of eosinophils suggest a relationship between eosinophil counts and pulmonary thrombus formation. In this study, to investigate the relationship of eosinophil counts with massive and submassive PE and also to reveal whether it can be used as a predictor.

\section{Materials and methods}

In this single-center study, we analyzed retrospectively 108 patients with $\mathrm{PE}$ and 75 healthy control subjects who were admitted to Bolu Abant Izzet Baysal University Hospital between January 2018 and July 2019. The study was approved by the Ethics committee of Bolu Abant Izzet Baysal University (Date and Decision no: 2020-168). Patients' demographic data such as age, weight, sex, height, heart rate, blood pressure, medication, comorbidity, smoking, and laboratory parameters were extracted from the electronic medical record. Hemogram values were obtained at initial presentation and after treatment.

Patients who had blood pressure $\geq 140$ / 90 $\mathrm{mmHg}$ or use antihypertensive drugs were considered as having hypertension (HT). Diabetes mellitus (DM) was determined by the current use of antidiabetic drugs, fasting blood glucose level $>126 \mathrm{mg} / \mathrm{dl}$, or HbAlc $\geq 7$. Those who had a total cholesterol level $\geq 200 \mathrm{mg} / \mathrm{dL}$, LDL-c level $\geq 130 \mathrm{mg} / \mathrm{dL}$, or use of cholesterollowering medication were considered as having hyperlipidemia (HL). An individual who was active smoker or had a smoking history of $>10$ packs per year was considered a smoker.

For diagnosing and determining the severity of PE, we leveraged current guidelines including symptoms, high D-dimer level, electrocardiogram, computed tomography pulmonary angiogram (CTPA), echocardiography, and positive cardiac enzymes [9]. Radiological data allowed us to distinguish between massive and sub-massive PE. From the radiological point of view, a massive $\mathrm{PE}$ is described as a reduction of lung perfusion in one lung (> 90\%) or total occlusion of a main pulmonary artery diagnosed with a CTPA. The remaining forms are described as sub-massive PE [10].

Patients were excluded from the study for the following reasons: pregnancy, systemic inflammatory or infectious disease, chronic obstructive pulmonary disease, any known hematological disease, hyper-eosinophilic syndrome, liver failure, and end-stage renal failure (eGFR $<15 \mathrm{ml} / \mathrm{min} / 1.73 \mathrm{~m}^{2}$ ). The study was approved by the local institutional board.

Samples of peripheral venous blood were gathered from the patients who were admitted with the diagnosis of pulmonary embolism. Levels of fasting plasma glucose, creatinine, high-density lipoprotein cholesterol, triglyceride, and low-density lipoprotein cholesterol were determined using an automatic biochemical analyzer (Architect C8000, USA). We measured complete blood counts using concurrent optical and impedance measurements (Cell Dyn 3700; Abbott Diagnostics, Lake Forest, Illinois, USA). Hematologic measurements on K3EDTAanticoagulated whole blood were performed using a hematologic analyzer.

\section{Statistical analysis}

We analyzed the data using SPSS 18.0 Statistical Package Software for Windows (SPSS Inc., Chicago, Illinois, USA). Quantitative and qualitative variables are expressed as mean \pm standard deviation (SD) and numbers and percentages. A one-way analysis of variance (ANOVA) was preferred for parameters with homogenous distribution, 
and post-hoc analyses were performed with Tukey's HSD. For parameters with nonuniform distribution or in case of inequality of variances, Kruskal-Wallis test served to compare variables across study subgroups. We used the Bonferroni-corrected Mann-Whitney $\mathrm{U}$ test for in-group differences. Variables with normal distribution were compared by T-test and expressed as mean $\pm \mathrm{SD}$. The Wilcoxon test was employed to assess the variations in eosinophil counts at the presentation and after the treatment in the pulmonary embolism group. The Spearman correlation analysis was used to assess the correlations between eosinophil counts and the right ventricle (RV) dysfunction on transthoracic echocardiogram or elevated cardiac troponin level with massive PE. A receiver operating curve (ROC) analysis served to find the predictive value of eosinophil count to distinguish between massive PE and sub-massive PE. All p-values of $<0.05$ were considered significant.

\section{Results}

The present study comprised a total of 183 subjects, the control group $(n=75)$, the submassive group ( $n=64)$, and the massive group $(n=44)$. All of the three groups showed similar baseline clinical characteristics and previous medications (Table 1). Regarding PE etiology, 15 patients (13.9\%) had cancer as an underlying disease; $32(29.6 \%)$ had a history of immobilization after an operation and 4 (3.7\%) had immobilization after an accident; 5 (4.6\%) had genetic predisposition; $3(2.8 \%)$ were in the postpartum period and $49(45.4 \%)$ had no predisposing factor.

Laboratory data other than those of white blood cells and eosinophil counts were similar between the groups (Table 2). The lowest eosinophil count was evident in the massive PE group whereas the control group had the highest value $(p<0.001)$. The massive group showed the highest value of white blood cell counts whereas the lowest value was evident in the

Table 1. General characteristics of the study population.

\begin{tabular}{|l|c|c|c|c|}
\hline Baseline characteristics & $\begin{array}{c}\text { Control group } \\
(\mathbf{n = 7 5})\end{array}$ & $\begin{array}{c}\text { Submassive group } \\
(\mathbf{n = 6 4 )}\end{array}$ & $\begin{array}{c}\text { Massive group } \\
(\mathbf{n = 4 4})\end{array}$ & $\boldsymbol{p}$ \\
\hline Age (mean \pm SD) (years)) & $59 \pm 7$ & $59 \pm 16$ & $63 \pm 16$ & 0.33 \\
\hline Body mass index (kg/m2) & $31 \pm 1$ & $30 \pm 2$ & $29 \pm 1$ & 0.24 \\
\hline Systolic blood pressure & $123 \pm 14$ & $118 \pm 12$ & $110 \pm 11$ & 0.09 \\
\hline Diastolic blood pressure & $74 \pm 9$ & $74 \pm 9$ & $71 \pm 11$ & 0.85 \\
\hline Male/female & $29 / 46$ & $22 / 42$ & $24 / 20$ & 0.10 \\
\hline Hypertension & $34(45 \%)$ & $31(48 \%)$ & $18(41 \%)$ & 0.74 \\
\hline Smoking & $16(21 \%)$ & $10(16 \%)$ & $10(23 \%)$ & 0.59 \\
\hline Diabetes mellitus & $16(21 \%)$ & $10(16 \%)$ & $8(18 \%)$ & 0.69 \\
\hline Acetyl salicylate & $23(31 \%)$ & $14(22 \%)$ & $8(18 \%)$ & 0.26 \\
\hline Calcium channel blocker & $15(20 \%)$ & $11(17 \%)$ & $7(16 \%)$ & 0.83 \\
\hline ACE inhibitor & $5(7 \%)$ & $9(14 \%)$ & $8(18 \%)$ & 0.14 \\
\hline ARB & $13(17 \%)$ & $11(17 \%)$ & $4(9 \%)$ & 0.42 \\
\hline B- blocker & $21(28 \%)$ & $15(23 \%)$ & $4(9 \%)$ & 0.05 \\
\hline
\end{tabular}

ACE: angiotensin-converting enzyme, ARB: angiotensin receptor blocker, SD: standard deviation. 
control group $(<0.001)$.

Kruskal-Wallis and Bonferroni-corrected Mann-Whitney U tests showed significantly different eosinophil counts between the massive PE and control groups ( $p<0.001)$, and between the massive and sub-massive PE groups $(p=0.005)$. It also tended to vary between the control and sub-massive PE groups $(\mathrm{p}=0.06)$.
A CT scanner revealed that mean RV to $\mathrm{LV}$ dimension ratios were significantly higher in the massive PE group than those in the submassive group (0.99 \pm 0.22 vs. $0.77 \pm 0.12$, $p<0.001$, respectively).

Spearman correlation test indicated that RV dysfunction or elevated cardiac troponin level was significantly correlated with the massive PE $(r=0.54, p<0.001)$; however, eosinophil

Table 2. Laboratory data of the study population.

\begin{tabular}{|c|c|c|c|c|}
\hline Laboratory data & $\begin{array}{l}\text { Control group } \\
(n=75)\end{array}$ & $\begin{array}{l}\text { Submassive group } \\
(n=64)\end{array}$ & $\begin{array}{l}\text { Massive group } \\
(n=44)\end{array}$ & $\mathbf{p}$ \\
\hline \multicolumn{5}{|c|}{ Median (Min-Max) } \\
\hline Creatinine (mg/dl) & $0.78(0.58-2.14)$ & $0.81(0.5-7.6)$ & $0.86(0.65-1.76)$ & 0.14 \\
\hline $\begin{array}{l}\text { Fasting plasma glucose } \\
(\mathrm{mg} / \mathrm{dl})\end{array}$ & $102(76-413)$ & $100(47-228)$ & $110(68-280)$ & 0.22 \\
\hline LDL-cholesterol (mg/dl) & $119(60-195)$ & $114(51-278)$ & $119(33-210)$ & 0.47 \\
\hline Triglyceride (mg/dl) & $133(37-212)$ & $131(48-294)$ & $145(45-338)$ & 0.12 \\
\hline Eosinophil counts $(\mathrm{K} / \mathrm{uL})$ & $0.174(0.003-0.853)$ & $0.121(0.002-0.620)$ & $0.050(0.003-0.383)$ & $<0.001$ \\
\hline Basophil counts $(\mathrm{K} / \mathrm{uL})$ & $0.065(0.010-0.580)$ & $0.064(0.007-0.183)$ & $0.059(0.008-0.288)$ & 0.68 \\
\hline \multicolumn{5}{|c|}{ Mean \pm S.D. } \\
\hline HDL-cholesterol (mg/dl) & $45 \pm 10$ & $47 \pm 12$ & $44 \pm 10$ & 0.28 \\
\hline Hemoglobin (g/dL) & $13.4 \pm 1.5$ & $12.6 \pm 1.9$ & $13.0 \pm 1.8$ & 0.14 \\
\hline Platelet counts $(\mathrm{K} / \mathrm{uL})$ & $240 \pm 60$ & $230 \pm 70$ & $225 \pm 75$ & 0.09 \\
\hline MPV (fL) & $8.1 \pm 1.5$ & $7.7 \pm 1.5$ & $7.5 \pm 1.2$ & 0.09 \\
\hline PDV (GSD) & $17.7 \pm 1.2$ & $17.7 \pm 1.4$ & $17.9 \pm 0.0 .9$ & 0.67 \\
\hline RDW (\%) & $16.5 \pm 1.9$ & $16.7 \pm 2.8$ & $16.7 \pm 2.3$ & 0.79 \\
\hline Monocyte counts (K/uL) & $0.52 \pm 0.19$ & $0.60 \pm 0.30$ & $0.60 \pm 0.19$ & 0.53 \\
\hline $\begin{array}{l}\text { Lymphocyte counts } \\
(\mathrm{K} / \mathrm{uL})\end{array}$ & $2.13 \pm 0.79$ & $1.95 \pm 0.86$ & $2.05 \pm 1.20$ & 0.11 \\
\hline WBC counts $(K / \mathbf{u L})$ & $7.35 \pm 1.63$ & $8.76 \pm 2.64$ & $9.19 \pm 3.98$ & 0.001 \\
\hline Total cholesterol (mg/dl) & $195 \pm 47$ & $200 \pm 38$ & $202 \pm 51$ & 0.72 \\
\hline
\end{tabular}

LDL: low-density lipoprotein, HDL: High-density lipoprotein, MPV: Mean platelet volume, PDW: Platelet distribution width, RDW: Red cell distribution width, WBC: White blood cell. 
count had an inverse correlation with the presence of massive PE $(r=-0.36, p<0.001)$.

A receiver operating curve (ROC) analysis yielded the sensitivity and specificity of eosinophil count for the discrimination of the massive PE group. At a cut-off value of $<0.125 \mathrm{u} / \mathrm{mm}^{3}$, the sensitivity and specificity for eosinophil count were $73 \%$ and $59 \%$, respectively, for determination of massive $\mathrm{PE}$ $(A U C=0.715,95 \%$ CI, 0.616-0.815) (Figure $1)$.

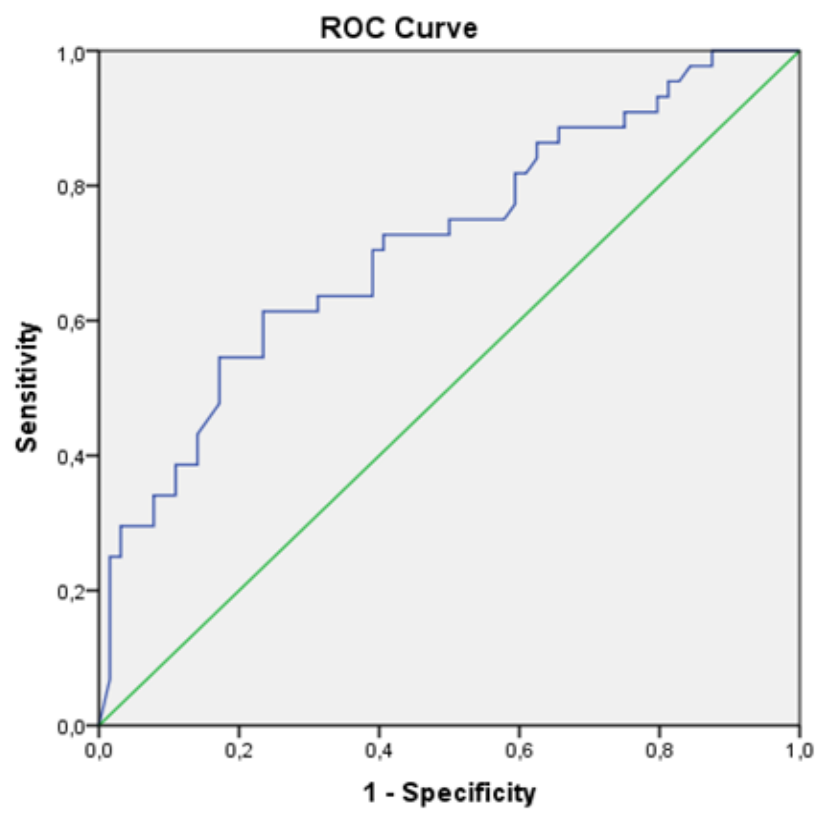

Figure 1. A receiver operating curve (ROC) analysis of eosinophil count for differentiating massive PE from sub-massive PE. At the cutoff value of $<0.125 \mathrm{u} / \mathrm{mm}^{3}$, sensitivity and specificity of eosinophil count were $73 \%$ and $59 \%$ for determination of massive PE respectively. $\quad(\mathrm{AUC}=0.715,95 \% \mathrm{CI}, 0.616$ 0.815). (AUC: area under the curve; CI: Confidence interval).

All patients in the sub-massive PE group received heparin therapy. As for the massive PE group, heparin treatment was administered in five patients with active cancer, twelve patients in the postoperative period, two patients in the post-accident immobilization period, and five patients who refused thrombolytic consent, and thus the remaining 20 patients received thrombolytic therapy. During six months of the follow-up, four patients $(3.6 \%)$ died either during the in-hospital stay (two patients) or during the follow-up after hospitalization (two patients).

The eosinophil count increased significantly, regardless of treatment modality, one week after the treatment compared to presentation with PE (0.112 (0.003-0.853) vs. (0.144 (0.011$0.914), p=0.01$, respectively).

\section{Discussion}

The association of decreased eosinophil count with the severity of PE was the principal finding of our study. It was significantly lower in the massive PE than the sub-massive PE and the control groups. As far as we know, this is the first study to show that a lower peripheral eosinophil count may support massive PE rather than sub-massive $\mathrm{PE}$ in patients with acute PE. Regarding the clinical aspect of this finding, decreased eosinophil counts should be a new therapeutic target in the massive PE subgroup.

Pulmonary embolism accounts for $10 \%$ of all causes of hospital deaths [11]. Patients with pulmonary embolism are clinically classified into massive and sub-massive patients based on blood pressure, right ventricular functions, high cardiac markers, and radiologically affected vascular bed [9].

It remains unclear how eosinophils are involved in the pathogenesis of thrombus formation. Neurotoxins, eosinophil cationic proteins, and major basic proteins released from eosinophils can cause endothelial damage resulting in fibrosis, thrombosis, and infarction [12]. Also, the enzyme peroxidase and the major basic protein released from eosinophilic granules may lead to thrombus formation by directly 
activating platelets and inhibiting thrombomodulin [13]. By means of another mechanism, tissue factors released from eosinophils bind to coagulation factor 7 and directly initiate the coagulation cascade [14]. The literature has reported the effects of eosinophils on thrombus formation in patients with the idiopathic hyper-eosinophilic syndrome, and the rare causes of arterial thrombosis and cardio-embolic stroke in childhood [1,15].

Coronary atherosclerotic plaques showed higher eosinophil concentration in the red thrombus, while the number of eosinophils in peripheral blood showed a negative correlation with the troponin count [13]. Furthermore, lower eosinophil counts were reported in myocardial infarction compared to those in unstable angina pectoris [16]. In addition, lower eosinophil counts were associated with a worse prognosis in patients undergoing percutaneous coronary intervention [17]. Mackman et al. investigated whether eosinophils were present in human coronary artery thrombus in patients with acute myocardial infarction of native vessels or stent thrombosis. They revealed that thrombi from female patients with previous stent thrombosis contained significantly elevated numbers of eosinophils. They also discussed which subgroups of patients could benefit most from an eosinophil-inhibition approach [18].

Therefore, a reverse correlation may exist between eosinophil counts and the burden of thrombi. However, eosinophil concentration has not been studied in aspirated PE thrombus. In light of the evidence that, for eosinophils, higher concentrations in coronary thrombi and less number in the systemic circulation are associated with the severity of acute coronary events, the fact that we detected fewer eosinophils in massive PE compared to sub- massive PE can be explained by the accumulation of eosinophils in the thrombus burden and thus their decreased number in the systemic circulation.

The present study showed that lower eosinophil counts may support the diagnosis of massive PE at acute presentation. This study also showed that eosinophil counts increased after treatment regardless of the treatment modality. Due to the retrospective nature of our study, the association of this finding with clinical outcomes is unclear. After conducting further studies on larger populations, these findings may prove useful both in the determination of massive PE and in the preference of treatment modalities including lytic therapy or mechanical interventions.

The main limitations of this study consist in the fact that it is a single-centered study with a retrospective design. Direct evidence was absent on the accumulation of eosinophils in the pulmonary emboli material. Another limitation lies in the fact that measurements were unavailable for the major basic protein, eosinophilic cationic protein, peroxidase, and other cytokine levels that may interfere with thrombosis. The retrospective design of the study did not allow us to determine cardiac enzymes, RV dysfunction data on transthoracic echocardiogram, and arterial blood gas parameters for all patients.

\section{Conclusion}

In conclusion, our study demonstrated an inverse relationship between the eosinophil count, a cheap and widespread hemogram parameter in peripheral blood, and the severity of PE. The counts were lower in massive PE than sub-massive PE. Therefore, lower eosinophil counts in an acute PE may lead physicians to suggest a higher probability of massive PE, and taking it as a predictor factor may require initiation of lytic therapy or 
mechanical interventions. This finding needs to be supported by larger, prospective, and multicenter studies.

Funding: The author(s) received no financial support for the research, authorship, and/or publication of this article.

Conflict of Interest: The authors declare that they have no conflict of interest.

\section{Ethical statement:}

The study was approved by Local Clinical Research Ethics Committee (Date and Decision Number: 2020-168), and written informed consent was obtained from each subject.

\section{Open Access Statement}

This is an open access journal which means that all content is freely available without charge to the user or his/her institution under the terms of the Creative Commons Attribution NonCommercial License (http://creativecommons.org/licenses/bync/4.0). Users are allowed to read, download, copy, distribute, print, search, or link to the full texts of the articles, without asking prior permission from the publisher or the author.

Copyright (c) 2021: Author (s).

\section{References}

[1]Li D, Xu L, Lin D, et al. Acute pulmonary embolism and deep vein thrombosis secondary to idiopathic hypereosinophilic syndrome. Respir Med Case Rep. 2018;25:213-215.

[2]Becattini C, Agnelli G. Aspirin for prevention and treatment of venous thromboembolism. Blood Rev. 2014;28(3):103-8.

[3]van der Hulle T, Tan M, den Exter PL, et al. Recurrence risk after anticoagulant treatment of limited duration for late, second venous thromboembolism. Haematologica. 2015;100(2):188-93.
[4]Prentice RL, Szatrowski TP, Fujikura T, et al. Leukocyte counts and coronary heart disease in a Japanese cohort. Am J Epidemiol. 1982;116(3):496-509.

[5]Jiang P, Wang DZ, Ren YL, et al. Significance of eosinophil accumulation in the thrombus and decrease in peripheral blood in patients with acute coronary syndrome. Coron Artery Dis. 2015;26(2):101-6.

[6]Sakai T, Inoue S, Matsuyama TA, et al. Eosinophils may be involved in thrombus growth in acute coronary syndrome. Int Heart J. 2009;50(3):267-77.

[7]Rohrbach MS, Wheatley CL, Slifman NR, et al. Activation of platelets by eosinophil granule proteins. J Exp Med. 1990;172(4):1271-74.

[8]Slungaard A, Vercellotti GM, Tran T, et al. Eosinophil cationic granule proteins impair thrombomodulin function. A potential mechanism for thromboembolism in hypereosinophilic heart disease. J Clin Invest. 1993;91(4):1721-30.

[9]Konstantinides SV, Torbicki A, Agnelli G, et al. 2014 ESC guidelines on the diagnosis and management of acute pulmonary embolism. Eur Heart J. 2014;35(43):303369, 3069a-3069k.

[10] Reekers JA, Baarslag HJ, Koolen MG, et al. Mechanical thrombectomy for early treatment of massive pulmonary embolism. Cardiovasc Intervent Radiol. 2003;26(3):246-50.

[11]Cushman M. Epidemiology and risk factors for venous thrombosis. Semin Hematol. 2007;44(2):62-69.

[12] Tenekecioglu E, Yilmaz M, Bekler A, et al. Eosinophil count is related with coronary thrombus in non ST-elevated acute coronary syndrome. Biomed Pap Med Fac Univ 
Palacky Olomouc Czech Repub. 2015;159(2):266-71.

[13] Mukai HY, Ninomiya $H$, Ohtani $K$, et al. Major basic protein binding to thrombomodulin potentially contributes to the thrombosis in patients with eosinophilia. Br J Haematol. 1995;90(4):892-99.

[14] Moosbauer C, Morgenstern E, Cuvelier SL, et al. Eosinophils are a major intravascular location for tissue factor storage and exposure. Blood. 2007;109(3):995-1002.

[15] Sharma SK, Kumar S, Seth T, et al. Eosinophilia: Rare cause of arterial thrombosis and cardioembolic stroke in childhood. World J Cardiol. 2012;4(4):1289.

[16] Sincer I, Gunes Y, Mansiroglu AK, et al. Differential value of eosinophil count in acute coronary syndrome among elderly patients. Aging Male. 2020;23(5):958-961.

[17]Toor IS, Jaumdally R, Lip GY, et al. Eosinophil count predicts mortality following percutaneous coronary intervention. Thromb Res. 2012;130(4):60711.

[18]Mackman N. Eosinophils, atherosclerosis, and thrombosis. Blood. 2019;134(21):17811782. 